\title{
Pevonedistat (MLN4924): mechanism of cell death induction and therapeutic potential in colorectal cancer
}

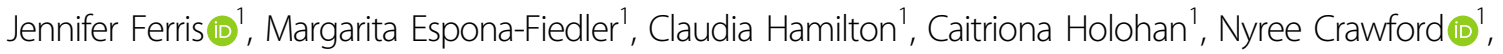 \\ Alex J. McIntyre', Jamie Z. Roberts (1)', Mark Wappett ${ }^{1}$, Simon S. McDade $\mathbb{D}^{1}$, Daniel B. Longley ${ }^{1}$ and Victoria Coyle ${ }^{1}$
}

\begin{abstract}
Pevonedistat (MLN4924), a selective inhibitor of the NEDD8-activating enzyme E1 regulatory subunit (NAE1), has demonstrated significant therapeutic potential in several malignancies. Although multiple mechanisms-of-action have been identified, how MLN4924 induces cell death and its potential as a combinatorial agent with standard-ofcare (SoC) chemotherapy in colorectal cancer (CRC) remains largely undefined. In an effort to understand MLN4924induced cell death in CRC, we identified p53 as an important mediator of the apoptotic response to MLN4924. We also identified roles for the extrinsic (TRAIL-R2/caspase-8) and intrinsic (BAX/BAK) apoptotic pathways in mediating the apoptotic effects of MLN4924 in CRC cells, as well as a role for BID, which modulates a cross-talk between these pathways. Depletion of the anti-apoptotic protein FLIP, which we identify as a novel mediator of resistance to MLN4924, enhanced apoptosis in a p53-, TRAIL-R2/DR5-, and caspase-8-dependent manner. Notably, TRAIL-R2 was involved in potentiating the apoptotic response to MLN4924 in the absence of FLIP, in a ligand-independent manner. Moreoever, when paired with SoC chemotherapies, MLN4924 demonstrated synergy with the irinotecan metabolite SN38. The cell death induced by MLN4924/SN38 combination was dependent on activation of mitochondria through BAX/BAK, but in a p53-independent manner, an important observation given the high frequency of TP53 mutation(s) in advanced CRC. These results uncover mechanisms of cell death induced by MLN4924 and suggest that this second-generation proteostasis-disrupting agent may have its most widespread activity in CRC, in combination with irinotecan-containing treatment regimens.
\end{abstract}

\section{Introduction}

By regulating protein turnover, the ubiquitin-proteasome system (UPS) plays a crucial role in regulating cellular proliferation and survival, with its inhibition regarded as an attractive anti-cancer strategy ${ }^{1}$. The first-generation proteasome inhibitor, Bortezomib, demonstrated efficacy in treatment of hematological malignancies; however, caused significant Grade 3/4 toxicities and demonstrated a lack of

\footnotetext{
Correspondence: Daniel B. Longley (d.longley@qub.ac.uk) or Victoria Coyle (v.coyle@qub.ac.uk)

${ }^{1}$ Centre for Cancer Research and Cell Biology, Queen's University Belfast, Belfast, Northern Ireland BT9 7BL, UK

These authors contributed equally: Daniel B. Longley, Victoria Coyle

Edited by Inna Lavrik
}

efficacy in solid tumors, as a single agent and in combination with standard-of-care therapies ${ }^{2}$. Secondgeneration proteasomal inhibitors, including more selective inhibitors, have since been developed with the goal of targeting the UPS in solid cancers. Foremost amongst these agents is Pevonedistat (MLN4924), which specifically targets NEDD8-activating enzyme E1 regulatory subunit (NAE1), which governs the first step of the enzymatic NEDDylation cascade. NEDD8 (neural precursor cell expressed developmentally downregulated protein 8 ) is a $9 \mathrm{kDa}$ ubiquitin-like protein that is conjugated to substrates in a similar manner to ubiquitin. NEDDylation is required for a number of cellular processes, the best characterized being activation of the

\section{(c) The Author(s) 2020}

(c) (i) Open Access This article is licensed under a Creative Commons Attribution 4.0 International License, which permits use, sharing, adaptation, distribution and reproduction cc) in any medium or format, as long as you give appropriate credit to the original author(s) and the source, provide a link to the Creative Commons license, and indicate if changes were made. The images or other third party material in this article are included in the article's Creative Commons license, unless indicated otherwise in a credit line to the material. If material is not included in the article's Creative Commons license and your intended use is not permitted by statutory regulation or exceeds the permitted use, you will need to obtain permission directly from the copyright holder. To view a copy of this license, visit http://creativecommons.org/licenses/by/4.0/. 
Cullin-RING E3 Ligase (CRL) family of E3 ubiquitin ligases $^{3,4}$, which are responsible for ubiquitination of $20 \%$ of the proteome.

MLN4924 forms an adduct between the C-terminus of NEDD8 and ATP-binding site of NAE1, resembling the NEDD8-adenylate intermediate formed during the NEDD8-NAE1 interaction ${ }^{5}$. MLN4924 is a potent inhibitor of NEDD8 conjugation, with the rapid deNEDDylation of Cullins- $1,-2,-3,-4 a,-4 b$, and -5 observed in vitro following treatment ${ }^{6}$, resulting in CRL inactivation and subsequent accumulation of CRL substrates ${ }^{7}$. Multiple studies have shown that MLN4924 induces cell death, promotes anti-tumor immunity ${ }^{8}$, and inhibits xenograft growth in various pre-clinical models of solid tumors ${ }^{9}$. MLN4924 has also demonstrated activity as monotherapy in clinical trials in hematological malignancies, and studies evaluating the combination of MLN4924 in combination with $\mathrm{SoC}$ chemotherapies in hematological malignancies, pediatric cancers, and lung cancer are ongoing ${ }^{10}$.

Colorectal cancer (CRC) has one of the highest Worldwide incidence and mortality rates, with $\sim 1.8$ million new cases and $\sim 881,000$ deaths occurring per annum $^{11,12}$. In metastatic CRC (mCRC), 5-year overall survival rates are dismal (only $\sim 8 \%$ of patients survive beyond 5 years $)^{13}$; thus, new therapeutic approaches are urgently needed. Previous pre-clinical studies in CRC have demonstrated that MLN4924 induces DNA rereplication, cell cycle arrest, and apoptosis in vitro, and impairment of patient-derived xenograft growth in vivo ${ }^{14}$. In this study, we assessed the potential clinical utility of MLN4924 using in vitro models of CRC, focusing on the mechanisms by which it induces cell death as a single agent, and its potential as a combinatorial agent with standard-of-care chemotherapeutics in genetically defined, clinically relevant disease subgroups.

\section{Results}

Wild-type p53 enhances MLN4924-induced CRC cell death

Although it has been demonstrated that MLN4924 has an impact on the apoptotic and DNA damage response machinery in cancer cells, the predominant effects of MLN4924 in CRC described to date relate to its effects on cell cycle progression ${ }^{15-17}$. Although it is evident from a number of studies that NEDDylation inhibition induces cancer cell death, with previous studies indicating a role of CRL substrate NOXA and the initiator caspase- $8^{18,19}$, the manner by which cell death occurs remains largely unresolved. Analysis of pharmacogenomic screening data from Version 2 of the Genomics of Drug Sensitivity in Cancer (GDSC) CRC cell lines indicated that p53 wildtype CRC cell lines $(n=12)$ were significantly more sensitive to MLN4924 than p53 mutant models $(n=30$; $p=0.019$; Fig. 1a). Notably, this was not observed in any other cancer types represented in the cell line panel other than cervical cancer $(p=0.04 ; n=7)$.

Western blot analyses of the effects of MLN4924 on PARP cleavage (a marker of cell death) confirmed that p53-proficient HCT116 and LoVo cells were significantly more sensitive to MLN4924-induced cell death than their respective isogenic p53-deficient daughter cell lines (Fig. 1b). Increased expression of both p53 and its canonical transcriptional target p21 was observed in response to MLN4924, and inhibition of NEDDylation was confirmed by loss of the NEDDylated form of Cullin-3 (Fig. 1b). The contribution of p53 in mediating sensitivity to MLN4924induced cell death was further confirmed by flow cytometry in both HCT116 and LoVo isogenic models (Fig. 1c). Moreover, MLN4924 significantly increased the activity of caspase- 8 , and also the executioner caspases of the apoptotic cascade, caspase-3/7, in a p53-dependent manner (Fig. 1d, e). Collectively, these results indicate that although p53-deficient CRC cells can undergo apoptosis in response to MLN4924, the level of cell death induced in p53 wildtype CRC cells is significantly greater.

\section{Mechanistic insights into MLN4924's mode-of-action from CCLE and GDSC databases}

To further investigate the mechanism-of-action of MLN4924 in CRC, we accessed baseline transcriptomics data from the Cancer Cell Line Encyclopedia (CCLE) ${ }^{20}$ and incorporated it with the cell-viability area under curve (AUC) values from the GDSC ${ }^{21,22}$ data, focusing on solid tumor cell lines. We noted that the density distribution of AUC values had a non-normal distribution (Fig. 2a) and so used the shoulder at AUC $=0.68$ to split the data into sensitive and resistant subgroups. The data were then filtered to include only the 35 CRC cell lines (Supplementary Table S1) with both a CCLE and a GDSC profile. Limma ${ }^{23}$ was used to compare the CRC cell lines divided by the 0.68 AUC threshold, identifying 948 genes that were differentially expressed between the two groups (Fold Change $[\mathrm{FC}]>2$; $p$-value $<0.05$; Fig. $2 \mathrm{~b}$ and Supplementary Table S2). EnrichR Pathway ${ }^{24}$ analysis of this gene set revealed significant signaling pathway differences in MLN4924-sensitive and -resistant models, with enrichment in adherens junction and adhesion in the more sensitive models (Fig. 2c and Supplementary Table S3). There were some compelling standalone markers of resistance (Supplementary Fig. 1A), including multi-drug resistance protein $1(A B C B 1)$ and Ras-related protein Rab-32 (RAB32), a marker of endoplasmic reticulum (ER)-, and mitochondrial stress. In terms of cell death signaling, the sensitive models were significantly more primed for caspase activation/apoptosis and TNF-related apoptosis-inducing ligand (TRAIL) signaling pathways; interconnected pathways which are transcriptionally regulated by $\mathrm{p} 53$. 


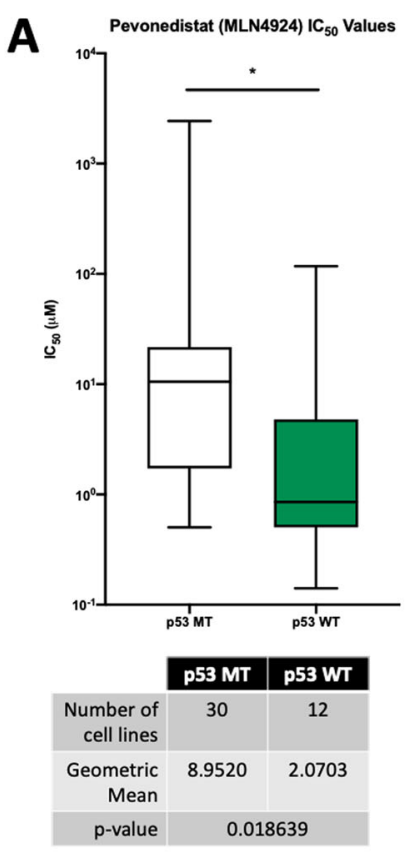

C

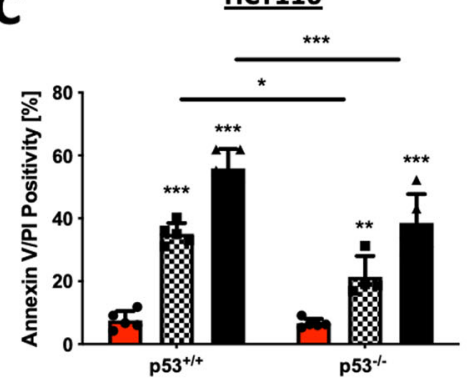

D

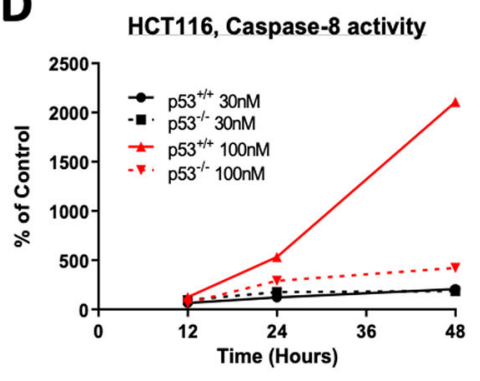

HCT116, Caspase-3/7 activity

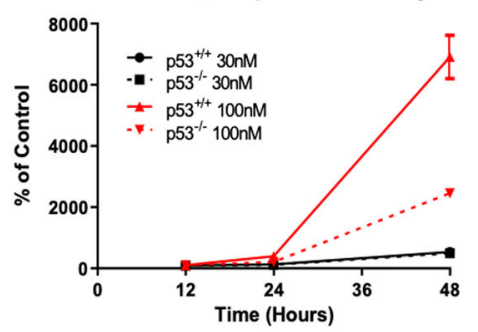

B $\underline{\mathrm{HCT} 116 \mathrm{p} 53^{+/+}} \quad \underline{\mathrm{HCT} 116 \mathrm{p} 53^{\%}}$

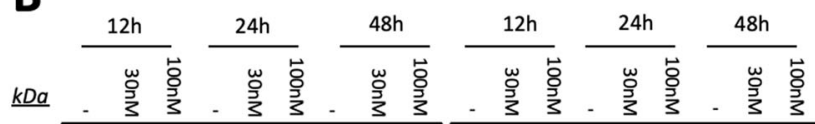

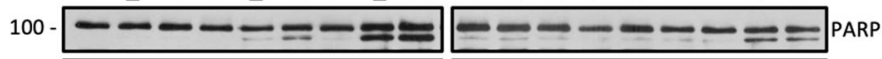

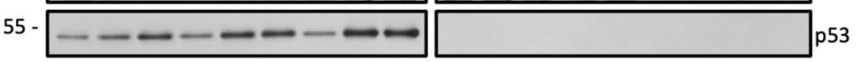

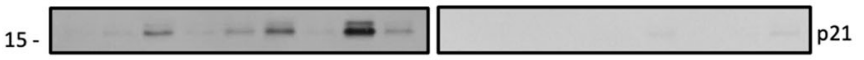

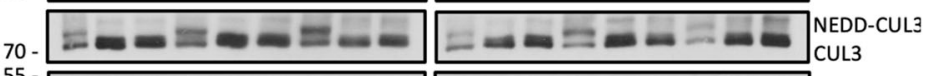

55 - - - -

LoVoshScr LoVoshp53

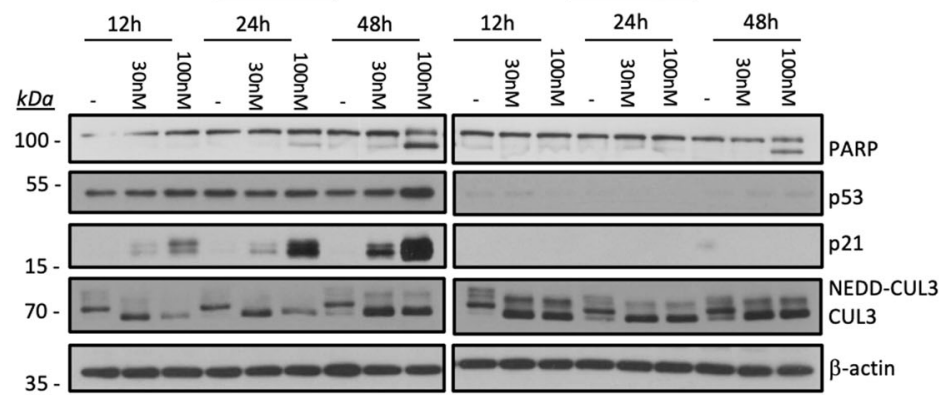

35

LoVo

$\square$ UT

30 30nM MLN4924

100nM MLN4924

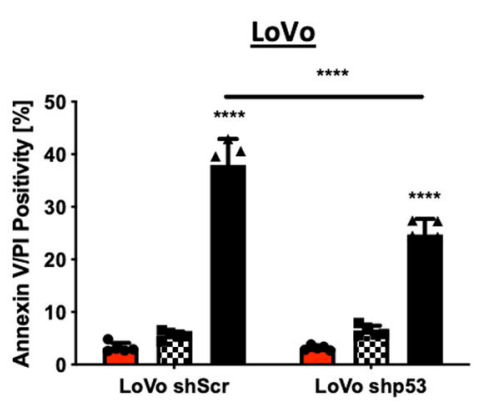

E

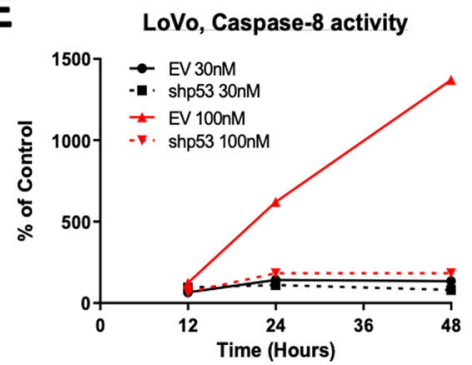

LoVo, Caspase-3/7 activity

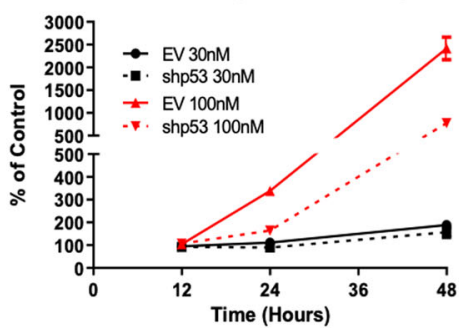

Fig. 1 (See legend on next page.) 
(see figure on previous page)

Fig. 1 p53 Proficiency enhances CRC cell death induced by MLN4924. a Sensitivity of a panel of p53 wild-type and mutant CRC cell lines to MLN4924. Data obtained from the Genomics of Drug Sensitivity in Cancer (GDSC) Project (https://www.cancerrxgene.org/). b Western blot analyses of poly-ADP ribose polymerase (PARP), p53, p21, Cullin-3 (CUL3), and $\beta$-actin in cells treated with 30 nM and 100 nM MLN4924 for 12, 24 and 48 h. c Annexin-V/Propidium lodide (AV/PI) flow cytometric analysis of HCT116 p53 $3^{+/+}$and $\mathrm{p} 53^{-/-}$, and LoVo shScr and shp53 cell lines treated for $48 \mathrm{~h}$ with $30 \mathrm{nM}$ and $100 \mathrm{nM}$ MLN4924. d, e Caspase-3/7 and -8 activity in HCT1 $16 \mathrm{p} 53^{+/+}$and p53 ${ }^{-/-}$, and LoVo shScr and shp53 cell lines treated with $30 \mathrm{nM}$ and $100 \mathrm{nM}$ MLN4924 for 12, 24, and $48 \mathrm{~h}$. An unpaired Student's T-test (Fig. 1a; GDSC dataset) or Two-way ANOVA multiple comparisons test was used to compute $p$-values for mean \pm SD of three independent experiments (Fig. 1c). Asterisks indicate statistically significant changes $\left({ }^{*} p \leq 0.05 ;\right.$ n.s denotes not significant).

\section{MLN4924 activates extrinsic and intrinsic apoptotic signaling pathway to elicit cell death in CRC}

To follow up the transcriptomics-based investigations, we next evaluated the effects of combining an $\mathrm{IC}_{50}(72 \mathrm{~h})$ dose of MLN4924 (30 nM) with a focused panel of small interfering RNAs (siRNAs) targeting established p53-dependent and -independent regulators of cell death in the HCT116 CRC cell line. Genes were ranked based on Sensitivity Index (SI) values, calculated from measurements of the effects on viability of siRNAs, MLN4924, and their combinations (Fig. $2 \mathrm{~d}$ ). To validate the screen, p53 was included and was indeed identified as the top mediator of sensitivity to MLN4924. In agreement with the pathway analyses, both procaspase-8 (CASP8) and TRAIL-R2/DR5 (TNFRSF1OB) were identified as mediators of response to MLN4924, along with procaspase-9 (CASP9) and NOXA (PMAIP1). Furthermore, RIPK1 (RIPK1), STAT3 (STAT3), cIAP1 (BIRC2), and FLICE-like inhibitory protein (FLIP; CFLAR) were identified as the top-ranked MLN4924 resistance genes (Fig. 2d), whose depletion increased the effects of MLN4924. Notably, three out of four of these proteins (RIPK1, cIAP1, and FLIP) are key components of TRAIL signaling, whereas caspase- 8 is the effector caspase for this pathway. Moreover, as demonstrated in Fig. 1d, MLN4924 significantly upregulated the activity of caspase8 , which correlated with an increase in caspase-3 activity (Fig. 1d; Spearman's $r=0.85 ; p=0.0009$ ). In agreement with this and the siRNA screen results, analysis of HCT116 cells, in which caspase-8 (CASP8) expression was knockedout with CRISPR-Cas9, identified a small inhibitory effect on MLN4924-induced apoptosis, as assessed quantitatively by flow cytometry (Fig. 2e) and qualitatively by PARP cleavage (Fig. 2f).

Procaspase-9, the initiator of the intrinsic mitochondrial apoptotic pathway, was also identified in our siRNA screen, along with the two main effectors of mitochondrial outer membrane permeabilization (MOMP), BAX and BAK, which lie upstream of procaspase-9 (Fig. 2d). The role of intrinsic (mitochondrial) apoptosis in mediating a cell death response to MLN4924 was confirmed by loss of mitochondrial membrane potential $(\Delta \Psi \mathrm{m})$ following $48 \mathrm{~h}$ MLN4924 treatment (Fig. 3a). The role of intrinsic apoptosis signaling in MLN4924-induced cell death was further assessed using a BAX/BAK double knockout (DKO) HCT116 daughter cell line, which was significantly more resistant to MLN4924-induced effects on cell viability $(<0.001$; Fig. $3 b)$ and cell death, as assessed by flow cytometry (Fig. 3c) and PARP cleavage (Fig. 3d). Moreover, in the matched EV HCT116 cells, both BAX and BAK were upregulated in response to MLN4924 (Fig. 3d). Activation of MOMP by BAX/BAK is triggered by the BH3-only protein subset of the Bcl-2 family, which either directly or indirectly promote BAX/BAK oligomerization in the outer mitochondrial membrane, leading to pore formation $^{25}$. The BH3-only proteins BIM and NOXA were both significantly upregulated in response to MLN4924 in a predominantly p53-dependent manner, although PUMA was downregulated. BID was also upregulated at $48 \mathrm{~h}$ in response to MLN4924 and a band corresponding to the MOMP-promoting form of BID (tBID) was also visible at this time point (Fig. 3e). We also found that MLN4924 significantly enhanced the expression of TRAIL-R2/DR5 in CRC cells, as well as Fas/CD95, both canonical p53 target genes (Fig. 3e and Supplementary Fig. 3A-C). Depleting either BID or NOXA with siRNA partially inhibited MLN4924-induced apoptosis; however, depleting either PUMA or BIM had no effect despite the latter's potent upregulation (Supplementary Fig. 3F, G). NOXA (PMAIP1) was also identified in the siRNA screen (Fig. 2d) and, although the effects of BID silencing were marginal in the siRNA screen, its importance is consistent with caspase- 8 activation identified in Fig. 2, as canonically, BID is activated by caspase- $8^{26}$. In support of this analysis, a BID CRISPRCas9-knockout model indicated that MLN4924-induced apoptosis was significantly abrogated in the absence of BID (Fig. 3f, g).

\section{FLIP downregulation enhances sensitivity to MLN4924 in a} p53-, caspase-8-, and TRAIL-R2-dependent manner

In the apoptosis-focused siRNA screen, downregulation of the Fas-Associated Via Death Domain (FADD)-binding partner and procaspase-8 paralog and regulator FLIP enhanced the effects of MLN4924, as did the known upstream regulators of FLIP expression RIPK1 and cIAP1 (via nuclear factor- $\mathrm{KB})^{27,28}$, and $\mathrm{STAT}^{29}$ (Fig. 2d). Consistent with this, siRNAs targeting both FLIP splice forms, FLIP-Long (FLIP(L)) and FLIP-Short (FLIP(S)), 

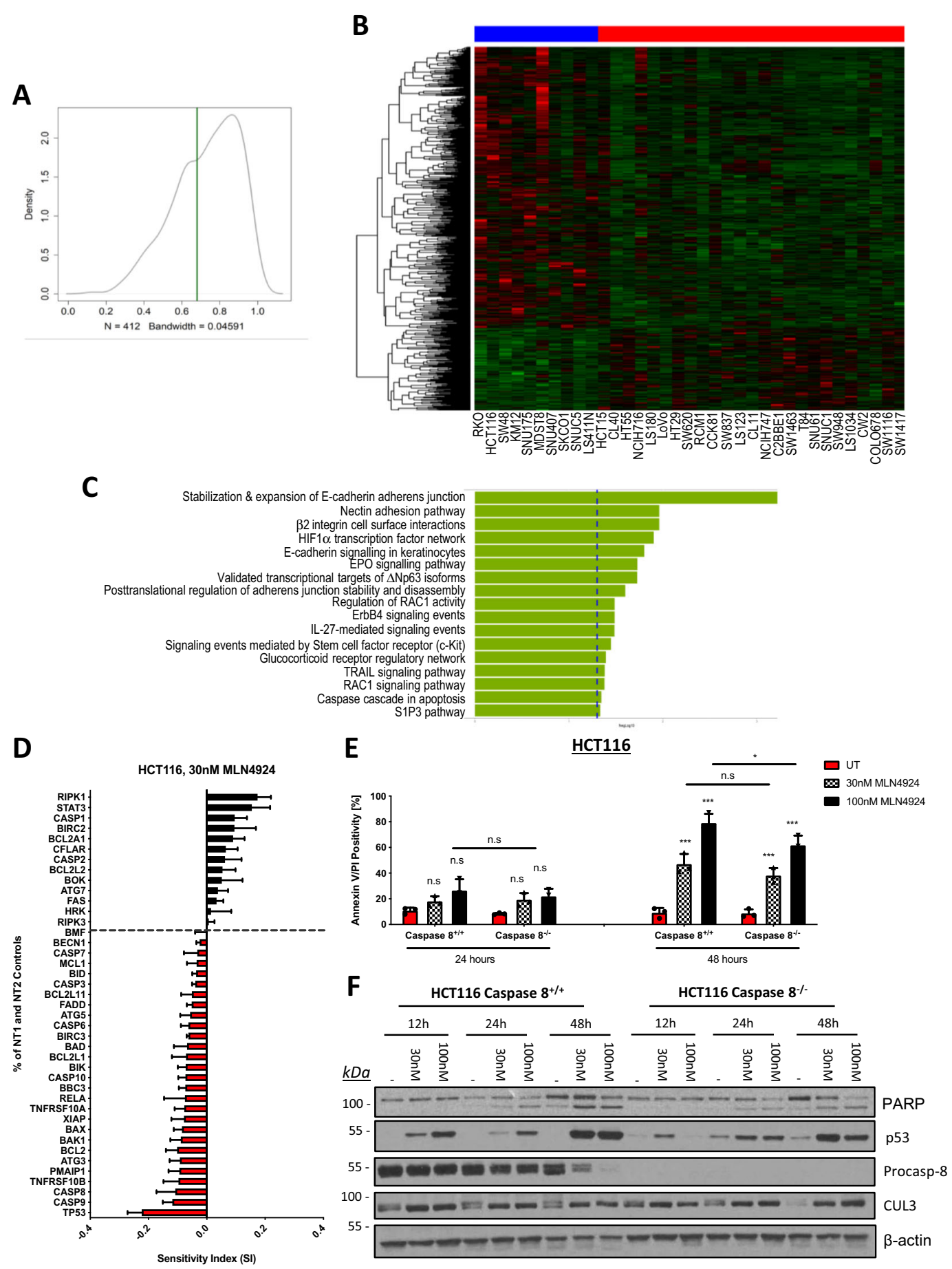

Fig. 2 Role of the extrinsic apoptotic pathway in mediating MLN4924-induced cell death. a Bimodal distribution of AUC values in solid cell lines from GDSC. $\mathbf{b}$ Nine hundred and forty-eight differentially expressed genes between 10 CRC cell lines with AUC $<0.68$ and 25 cell lines with AUC $>0.68$. c Pathway analysis of 948 genes from b. d HCT116 cells were reverse transfected with $10 \mathrm{nM}$ siRNA targeting known regulators of cell death prior to treatment with $30 \mathrm{nM}$ MLN4924. Viability was assessed after $72 \mathrm{~h}$. Sensitivity Index (SI) was used to determine which siRNAs enhance or antagonize the effects of MLN4924, with positive values indicating a sensitizing effect and negative values an antagonistic effect. e Annexin-V/PI flow cytometric analyses of HCT116 Caspase-8 CRISPR knockout and matched Empty Vector (EV; Caspase- $8^{+/+}$) cell lines treated for 24 and $48 \mathrm{~h}$ with 30 and $100 \mathrm{nM} \mathrm{MLN4924.}$ f Western blot analysis of PARP, p53, caspase-8, CUL3, and $\beta$-actin following 24 and $48 \mathrm{~h}$ treatment with 30 and $100 \mathrm{nM}$ MLN4924 in the HCT116 Caspase-8 CRISPR knockout and EV control cell lines. Two-way ANOVA with multiple comparisons test was used to compute $p$-values for mean \pm SD of at least three independent experiments. Asterisks indicate statistically significant changes $\left({ }^{*} p \leq 0.05,{ }^{* *} p \leq 0.01\right.$; n.s denotes not significant). 


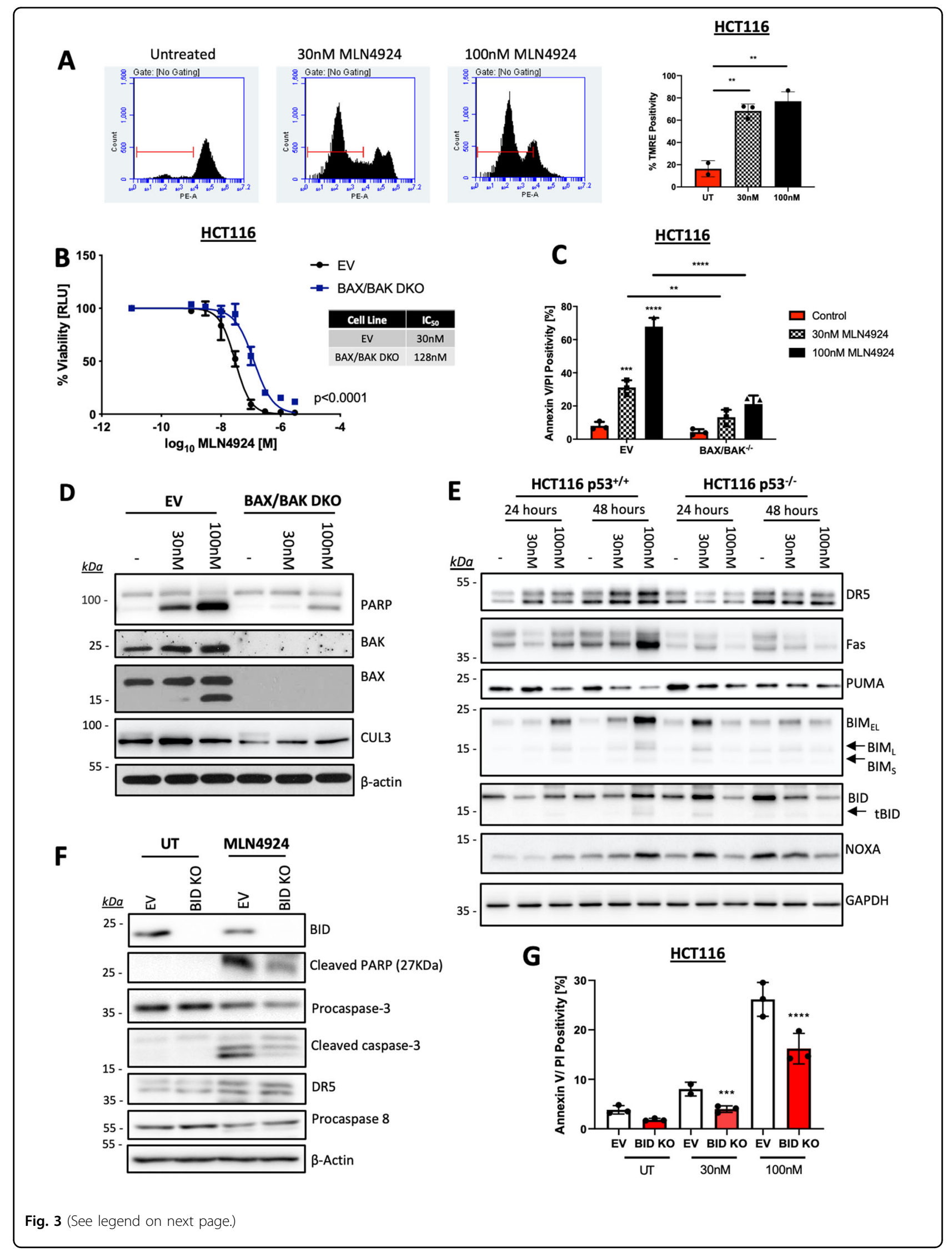


(see figure on previous page)

Fig. 3 MLN4924 activates the intrinsic apoptotic signaling pathway to elicit cell death. a Mitochondrial outer membrane potential ( $\triangle \psi m$ ) was measured following a $48 \mathrm{~h}$ treatment with $30 \mathrm{nM}$ or $100 \mathrm{nM}$ MLN4924 via flow cytometry following TMRE staining (25 nM). b CellTiter-Glo ${ }^{\circledR}$ cell viability assay in control (EV) and HCT116 BAX/BAK double-knockout (DKO) cells treated for $72 \mathrm{~h}$ with MLN4924. Values were normalized to untreated control. $I_{50}$ values were calculated via a nonlinear regression model, based on the mean \pm SD of three independent experiments. $\mathbf{c}$ Annexin-V/PI

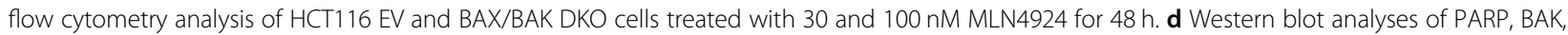

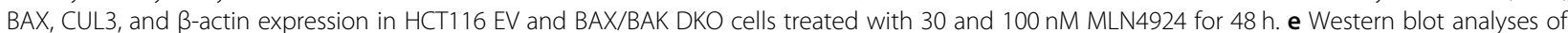

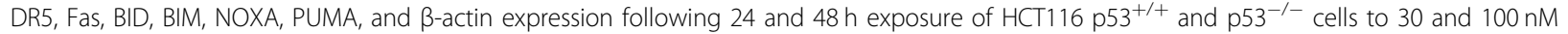
MLN4924. f Western blot analyses of BID, PARP, caspase-3, DR5, procaspase-8, and $\beta$-actin following treatment of HCT116 EV BID CRISPR KO cells treated with $100 \mathrm{nM}$ MLN4924 for $48 \mathrm{~h}$. g High-content annexin-V/PI analysis of HCT116 EV and BID knockout (KO) cells following treatment with MLN4924 for $48 \mathrm{~h}$. An unpaired Student's T-test (Fig. 3a) or Two-way ANOVA with multiple comparisons test was used to compute $p$-values for mean \pm SD of three independent experiments. Asterisks indicate statistically significant changes $\left({ }^{*} p \leq 0.05,{ }^{* *} p \leq 0.01\right.$, and ${ }^{* * *} p \leq 0.001$; n.s denotes not significant).

significantly enhanced MLN4924-induced apoptosis, but only in the HCT116 p53 wild-type model (Fig. 4a), an effect also observed in the LoVo paired cell lines (Supplementary Fig. 2A). Protein levels of FLIP(S) were downregulated in response to MLN4924 in both p53proficient and -deficient models; FLIP(L) downregulation was also observed, but only in the p53-deficient models (Fig. 4b and Supplementary Fig. 2B). The downregulation of FLIP(S) in response to MLN4924 may explain why specific downregulation of $\operatorname{FLIP}(\mathrm{L})$ was sufficient to enhance MLN4924-induced apoptosis in the p53 wildtype models, particularly in the HCT116 model, where MLN4924-induced FLIP(S) downregulation was more profound (Fig. 4a, b and Supplementary Fig. 2A, B). Caspase-3/7 activity was in line with the cell death induced in response to co-treatment with siFLIP and MLN4924 (Fig. $4 \mathrm{c}$ and Supplementary Fig. 2C). Notably, enhancement of apoptosis induced by FLIP depletion in combination with MLN4924 was clearly caspase-8-dependent, as apoptosis induction and PARP cleavage were abrogated in the caspase-8-deficient daughter cell line (Fig. 4d, e).

The upstream regulator of caspase-8-mediated apoptosis, TRAIL-R2 (DR5, TNFRSF1OB), was also identified in the pathway analysis (Fig. 2c) and siRNA screen as a potential mediator of response to MLN4924 (Fig. 2d). Moreover, MLN4924 treatment led to upregulation of DR5 expression (Fig. 3e and Supplementary Fig. 3A). In HCT116 cells, siRNA-mediated TRAIL-R2 downregulation abrogated PARP cleavage induced by MLN4924 alone (Fig. 4f), indicating a role for the receptor in mediating MLN4924induced apoptosis; no PARP cleavage was observed in response to MLN4924 alone in the LoVo model (which is also more resistant in the GDSC panel) at this concentration and time point (Supplementary Fig. 3C). However, in both cell lines, the enhanced cell death induced by MLN4924 in FLIP-depleted cells was also TRAIL-R2dependent (Fig. 4f, g and Supplementary Fig. 3C, D). Notably, however, these effects were not dependent on TRAIL-R2 ligation, as the cell death induced by MLN4924 in FLIP-depleted cells was not rescued by co-treatment with a TRAIL-neutralizing antibody (Fig. 4h and Supplementary Fig. 3E). Collectively, these results indicate that when FLIPmediated inhibition is overcome, MLN4924 activates p53dependent, TRAIL-R2-, and caspase-8-mediated apoptosis in a ligand-independent manner.

\section{MLN4924 exhibits p53-independent synergy with SN38}

The above data indicate that MLN4924 can prime the mitochondria for apoptosis by upregulating the levels of NOXA, BAX, BAK, and BID. Moreover, the importance of wild-type p53 in mediating the apoptotic effects of MLN4924 is consistent with its role as a transcriptional activator of the genes encoding NOXA, BAX, and TRAIL$\mathrm{R} 2^{30-32}$. As inefficient apoptosis induction is a major cause of chemotherapy resistance ${ }^{33}$, we hypothesized that MLN4924 may thereby enhance the effectiveness of standard-of-care CRC chemotherapeutic agents in a p53dependent manner. Thus, we performed a series of clonogenic survival assays to determine the efficacy of MLN4924 in combination with the backbone chemotherapeutic agent for CRC, 5-fluorouracil (5FU), and in combination with the two other main chemotherapeutics used to treat this disease, oxaliplatin and irinotecan. Given the role of p53 in mediating the effects of single-agent MLN4924, we assessed activity in p53 wild-type and p53deficient HCT116 models. Surprisingly, there was no significant synergy between MLN4924 and either 5FU or oxaliplatin regardless of the p53 status in HCT116 cells (Supplementary Fig. 4A-F). However, there was a clear interaction between MLN4924 and the active metabolite of irinotecan, SN38, at the lowest concentrations used (Fig. 5a); notably, this effect was clearly p53-independent. These results were confirmed by Western blot analyses, AV/PI flow cytometry, and caspase activity assays (Fig. 5b-d), and similar results were obtained in the LoVo model (Fig. 5e), although significant p53-independent enhancement of 5FU-induced apoptosis and p53-dependent enhancement of oxaliplatin-induced apoptosis in MLN4924 co-treated cells were also observed in this cell line (Supplementary Fig. 5A, B). 


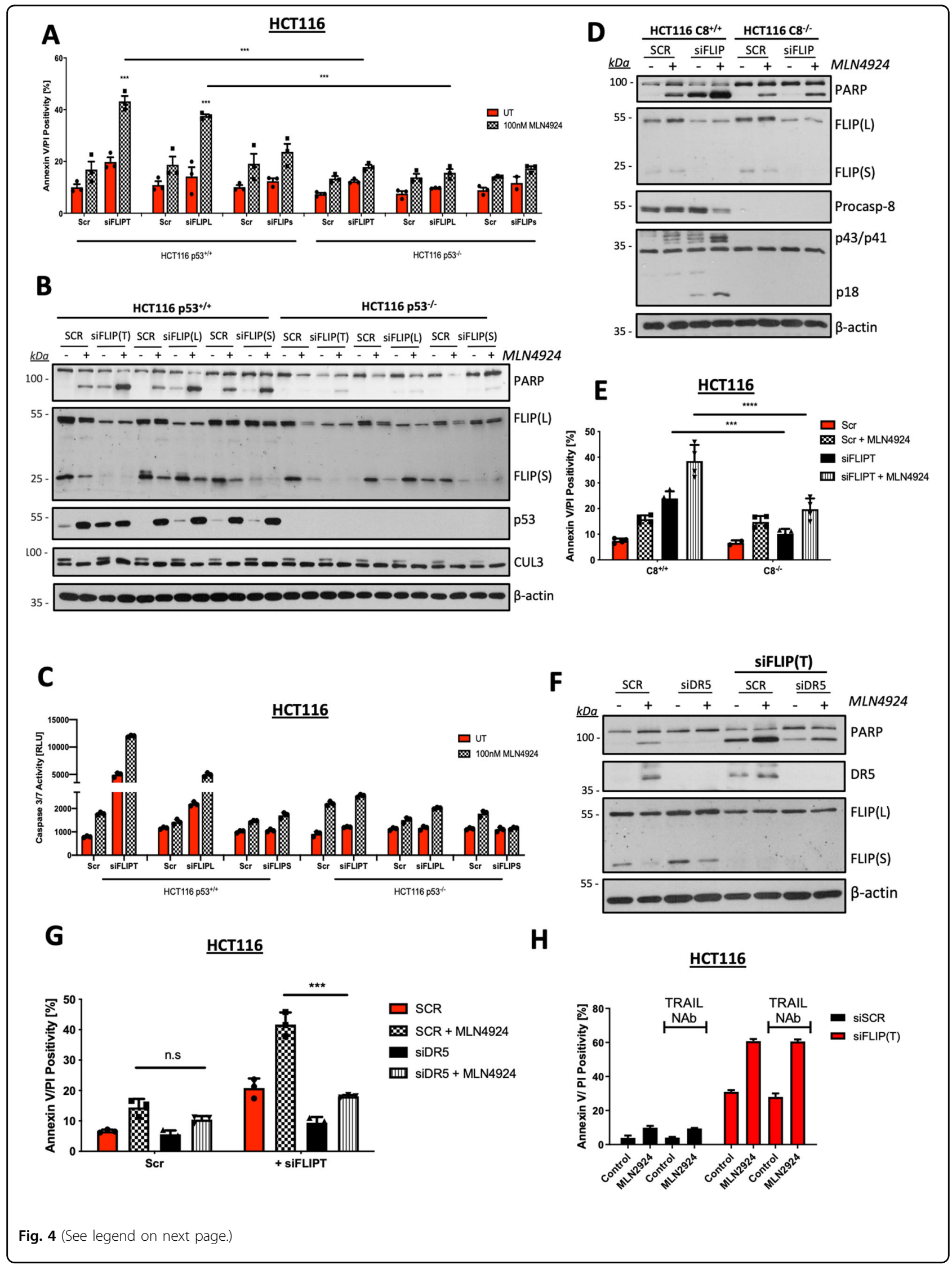


(see figure on previous page)

Fig. 4 FLIP downregulation enhances sensitivity to MLN4924 in a p53-, caspase-8-, and TRAIL-R2-dependent manner. a Annexin-V/PI flow cytometry analysis of HCT116 $553^{+/+}$and $\mathrm{p} 53^{-/-}$cell lines pre-treated with $10 \mathrm{nM}$ scrambled control (SCR) siRNA, FLIP siRNA targeting both FLIP(L) and FLIP(S) (siFLIP(T)), FLIP(L)-specific siRNA (siFLIP(L)), and FLIP(S)-specific siRNA (siFLIP(S)) for $6 \mathrm{~h}$, followed by $24 \mathrm{~h}$ treatment with $100 \mathrm{nM}$ MLN4924. b Western blot analyses of PARP, FLIP, p53, CUL3, and $\beta$-actin following transfection of HCT116 p53 $3^{+/+}$and $\mathrm{p} 53^{-/-}$cell lines with $10 \mathrm{nM}$ SCR, siFLIP (T), siFLIP(L), or siFLIP(S) siRNAs and $24 \mathrm{~h}$ treatment with $100 \mathrm{nM}$ MLN4924. c Caspase-3/7 activity in HCT116 cells co-treated with $10 \mathrm{nM} \mathrm{SCR}$, siFLIP(T), siFLIP(L), or siFLIP(S) siRNAs $100 \mathrm{nM}$ MLN4924 for $24 \mathrm{~h}$. d Western blot analyses of PARP, FLIP, Caspase-8, CUL3, and $\beta$-actin following transfection of HCT116 CASP8 $(\mathrm{C} 8)^{+/+}$and CASP8 (C8) $)^{-/-}$cell lines with $10 \mathrm{nM}$ SCR or siFLIP(T) siRNAs and $24 \mathrm{~h}$ treatment with $100 \mathrm{nM}$ MLN4924. e Flow cytometry analysis of apoptosis in CASP8 (C8) $)^{+/+}$and CASP8 (C8) ${ }^{-/-}$cell lines transfected with $10 \mathrm{nM}$ SCR or siFLIP(T) siRNAs and treated with $100 \mathrm{nM}$ MLN4924 for $24 \mathrm{~h}$. f Western blot analyses of PARP, DR5 (TRAIL-R2), FLIP, and $\beta$-actin expression in HCT116 parental cell lines. Cells were pre-treated with DR5specific siRNA for $24 \mathrm{~h}$, reseeded, and transfected with $10 \mathrm{nM}$ SCR or siFLIP(T) prior to $24 \mathrm{~h}$ treatment with $100 \mathrm{nM}$ MLN4924. g Annexin-V/PI flow cytometry analysis of HCT116 cells pre-treated with control (SCR) or DR5-specific siRNA (siDR5) for $24 \mathrm{~h}$, reseeded, and transfected with SCR or siFLIP (T) siRNAs prior to $24 \mathrm{~h}$ treatment with $100 \mathrm{nM}$ MLN4924. $\mathbf{h}$ High-content annexin-V/PI analysis of HCT116 cells transfected with $10 \mathrm{nM}$ FLIP(T)-specific siRNA and treated with $100 \mathrm{ng} / \mathrm{mL}$ of TRAlL-neutralizing antibody $\pm 100 \mathrm{nM} \mathrm{MLN4924}$ for $24 \mathrm{~h}$. Two-way ANOVA with multiple comparisons test was used to compute $p$-values for mean \pm SD of three independent experiments. Asterisks indicate statistically significant changes $\left({ }^{*} p \leq 0.05,{ }^{* *} p \leq 0.01\right.$, and ${ }^{* * *} p \leq 0.001$; n.s denotes not significant).

The interaction between MLN4924 and SN38 was all the more notable as, similar to MLN4924, the effects of SN38 in a wider panel of CRC models was also p53dependent, with p53 wild-type models significantly more sensitive (Supplementary Fig. 5C). To investigate the mechanism of action behind this synergy, we assessed the impact of the combination therapy in BAX/BAK- and BID-deficient models. A lack of BAX/BAK almost completely attenuated apoptosis induction (Fig. 5f, g), indicating that the combination drives cell death primarily via the intrinsic apoptosis pathway.

\section{Discussion}

Essential for cellular homeostasis, controlled protein turnover is critical for most, if not all, biological processes, including cell cycle progression, cell death, gene expression, and signal transduction. The UPS regulates turnover of the majority of intracellular proteins via interplay between E1 ubiquitin-activating enzymes, E2 ubiquitin-conjugating enzymes, and E3 ubiquitin ligases, which catalyze the transfer of the posttranslational modifier, ubiquitin, onto substrate proteins. The functionality of the largest family of E3 ligases, the CRLs, is controlled by the ubiquitin-like modifier, NEDD8, with NEDDylation of Cullin proteins required to activate the CRL complex. Similar to the ubiquitin-conjugation cascade, NEDD8 conjugation involves the interplay of three enzymes (E1, E2, and E3).

Recognition that the process of malignant transformation profoundly affects protein homeostasis has led to the development of therapeutics that exploit tumor-selective vulnerability to the disruption of proteostasis. Although approved for the treatment of multiple myeloma, the proteasomal inhibitor Bortezomib has shown little anti-tumor efficacy in solid tumors and causes substantial toxicities. The development of more selective, targeted inhibitors of protein degradation aims to reduce the side effects associated with global proteasomal inhibition. MLN4924 is currently undergoing Phase I and Phase II clinical trials in hematological malignancies and some solid tumors (e.g., non-small cell lung carcinoma, melanoma, liver cancer, and mesothelioma), both as a single agent and in combination with chemotherapies, including platinums and taxanes ${ }^{10}$.

A growing number of studies have demonstrated MLN4924's potential value in the treatment of CRC. For example, Zhang et al. ${ }^{34}$ observed that MLN4924 was a potent radio-sensitizing agent in vitro, enhancing G2/M arrest and apoptosis in HT29 and HCT116 CRC cell lines. This effect was rescued by p27 knockdown, suggesting that this well-established CRL cell cycle regulatory protein is involved in mediating the impact of MLN4924 in response to radiation in these models. A single-agent study in 2009 by Soucy et al. ${ }^{9}$ discovered that MLN4924 deregulated S-phase DNA synthesis, promoted accumulation of DNA damage, and subsequently activated apoptosis in HCT116 cells. The inhibitor was also established to be well tolerated in vivo and caused potent xenograft growth inhibition ${ }^{9}$. Further study demonstrated that the disruption of DNA synthesis induced by MLN4924 treatment was due to stabilization of the CRL substrate Cdt1, a DNA replication factor that induces DNA re-replication upon MLN4924 exposure ${ }^{35}$. Moreover, Picco et al. ${ }^{14}$ investigated response to MLN4924 in 122 CRC cell lines and identified a transcriptional signature of sensitivity. This signature was able to predict response to treatment in patient-derived xenograft models; however, the response observed was a stabilization of tumor growth rather than a reduction ${ }^{14}$, suggesting that MLN4924 may be most effective in combination with other agents as a treatment strategy for CRC.

MLN4924 has been shown to stabilize p53 through its capacity to induce ribosomal stress; e.g., inhibition of RPL11 NEDDylation enables its translocation from ribosomes to impede the MDM2-p53 interaction, leading to p53 stabilization ${ }^{36}$. Indeed, we observed p53 stabilization 
A

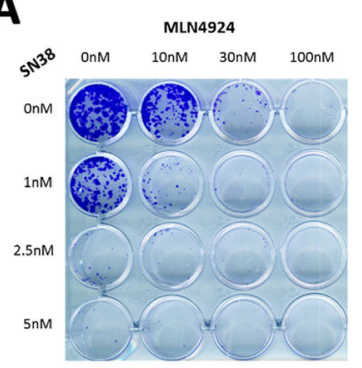

HCT116 p53+/+

C

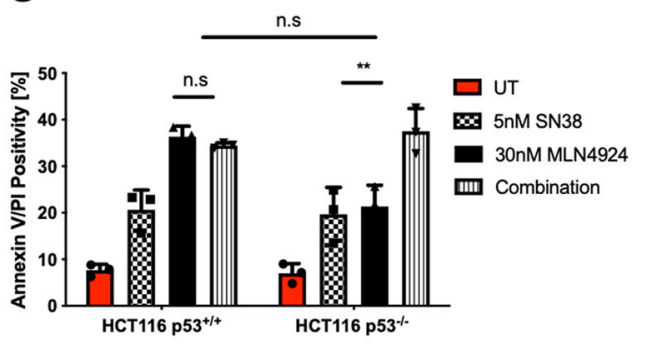

$\mathbf{E}$

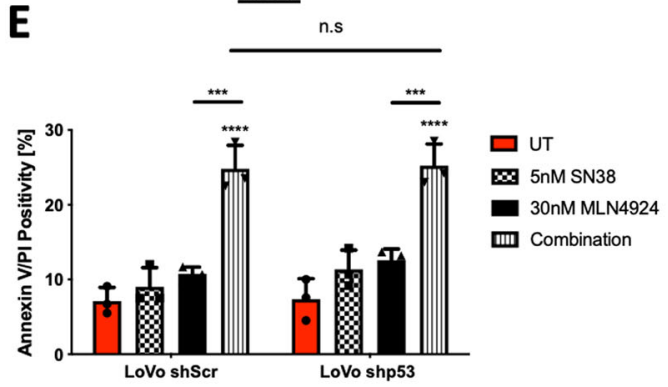

B $\quad$ HCT116

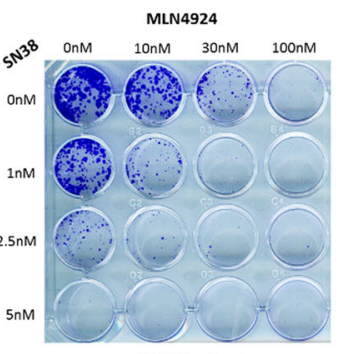

HCT116 $\mathrm{p} 53 \%$

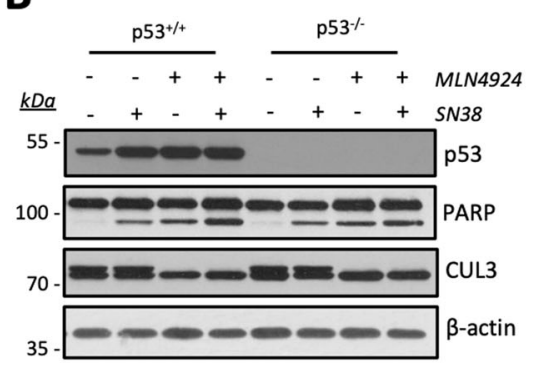

HCT116
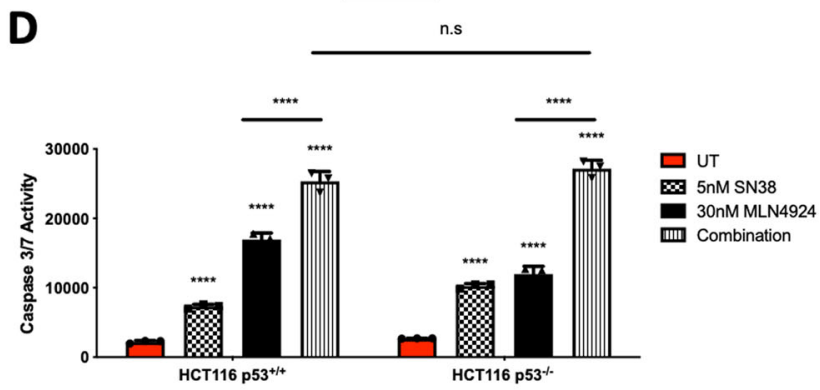

$\mathbf{F}$

HCT116

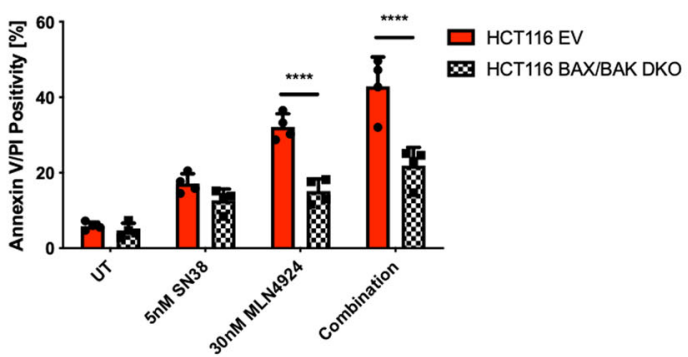

HCT116

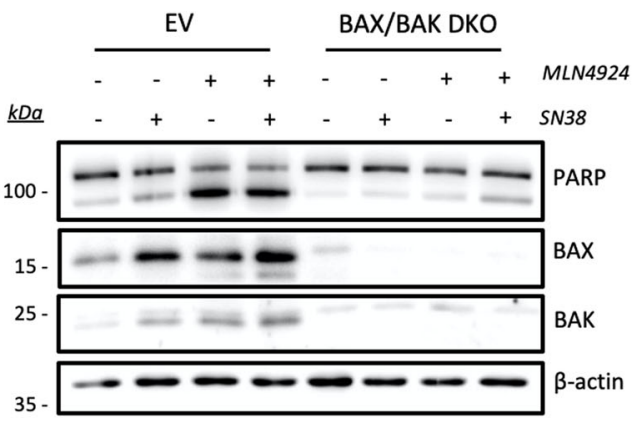

Fig. 5 (See legend on next page.)

in response to MLN4924 and found that p53-deficient CRC models were more resistant to MLN4924-induced apoptosis, although significant cell death was still observed in p53-deficient models. This was supported by data from the GDSC online database, which indicated that p53 wild-type CRC models were overall significantly more 
(see figure on previous page)

Fig. 5 MLN4924 exhibits p53-independent synergy with SN38. a Clonogenic assay in HCT116 p53 $3^{+/+}$and p53 ${ }^{-/-}$cells co-treated with MLN4924 and SN38 for $24 \mathrm{~h}$ prior to media replenishment. Colonies were allowed to form for 7 days prior to fixing and staining with Crystal Violet. b Western blot analysis of p53, PARP, CUL3, and $\beta$-actin following $48 \mathrm{~h}$ treatment of HCT116 p53 $3^{+/+}$and p53 ${ }^{-/-}$cells with $5 \mathrm{nM}$ SN38 and $30 \mathrm{nM}$ MLN4924, single agent, and in combination. c Annexin-V/PI flow cytometric analysis of HCT116 p53 $3^{+/+}$and p $53^{-/-}$cell lines treated for $48 \mathrm{~h}$ with $5 \mathrm{nM}$ SN38 and $30 \mathrm{nM}$ MLN4924 alone or in combination. d Caspase-3/7 activity in HCT116 p53 $3^{+/+}$and p53 $3^{-/-}$cells treated with $5 \mathrm{nM} \mathrm{SN38,} 30 \mathrm{nM}$ MLN4924, or a combination of both compounds for $48 \mathrm{~h}$. e Annexin-V/PI flow cytometric analysis of LoVo shScr and shp53 cell lines treated for $48 \mathrm{~h}$ with $5 \mathrm{nM}$ SN38 and 30 nM MLN4924 alone or in combination. f Annexin-V/PI flow cytometric analysis of HCT116 BAX/BAK CRISPR-knockout and matched Empty Vector (EV) cell lines treated for $48 \mathrm{~h}$ with $5 \mathrm{nM}$ SN38 and $30 \mathrm{nM}$ MLN4924 alone or in combination. $\mathbf{g}$ Western blot analysis of PARP, BAX, BAK, and $\beta$-actin in the HCT116 BAX/BAK CRISPR-knockout and matched EV cell lines treated for $48 \mathrm{~h}$ with $5 \mathrm{nM}$ SN38 and $30 \mathrm{nM}$ MLN4924 alone or in combination. Two-way ANOVA with multiple comparisons test was used to compute $p$-values for mean \pm SD of three independent experiments. Asterisks indicate statistically significant changes $\left({ }^{*} p \leq 0.05,{ }^{* *} p \leq 0.01\right.$, and ${ }^{* * *} p \leq 0.001$; n.s denotes not significant).

sensitive to MLN4924 than p53 mutant models. Notably, this was also the case in only one other tumor type (cervical cancer), suggesting that p53's role in mediating the cytotoxic effects of MLN4924 is tumor type-dependent.

NOXA has previously been reported to mediate MLN4924-induced cell death ${ }^{18,37}$ and our results (Supplementary Fig. 3F, G) would support this. However, we also found that another BH3-only protein BID plays an important role in mediating MLN4924-induced apoptosis in CRC cells. BID is activated downstream of caspase- 8 in the extrinsic apoptotic pathway; consistent with this, we identified a role for caspase- 8 in mediating cell death in response to MLN4924 alone. This is in agreement with a study in esophageal cancer, which demonstrated the interplay between ER stress and apoptosis in response to MLN4924. This study found that ATF4 was stabilized upon MLN4924 treatment, which subsequently activated the ER stress mediator CCAAT-enhancer-binding protein homologous protein (CHOP), leading to the upregulation of the death receptor TRAIL-R2 ${ }^{19}$ and activation of caspase- 8 and BID. Consistent with the roles of NOXA and BID, we found that co-deletion of BAX and BAK, both of which were upregulated in response to MLN4924, protected CRC cells from MLN4924-induced cell death, indicating an important role for intrinsic apoptosis in this response. With regard to p53-mediated sensitivity, the transcription factor is also capable of transcriptionally activating BAX, as well as directly activating $\mathrm{BAX}$ and $\mathrm{BAK}$ at the mitochondrial outer membrane $^{31,38,39}$ to initiate MOMP.

FLIP is a well-established apoptosis-regulatory protein implicated in resistance to standard-of-care therapies ${ }^{40-42}$, regulating procaspase- 8 processing at the DISC and related complexes in a splice form-specific manner ${ }^{43}$. FLIP silencing in combination with short-term MLN4924 treatment significantly enhanced cell death in a manner that was highly dependent on p53, TRAIL-R2, and caspase-8. Moreover, MLN4924 upregulated TRAIL-R2 expression in both p53 wild-type models (HCT116 and LoVo). These results indicate that FLIP silencing induces p53-dependent,
TRAIL-R2-, and caspase-8-mediated apoptosis upon treatment with MLN4924. However, ligation of TRAIL-R2 by its canonical ligand TRAIL was not required. TRAILR2-dependent and TRAIL-independent cell deaths in response to chemotherapy ${ }^{44}$ and during ER stress ${ }^{45}$ have been described, with the latter shown to be triggered by binding of misfolded proteins to TRAIL-R2 at the ER ${ }^{46}$, a result consistent with the reported impact of MLN4924 on ER stress (and consistent with our CCLE/GDSC analyses). At the protein level, MLN4924 treatment downregulated FLIP(S) in both p53-proficient and -deficient models, but only downregulated FLIP(L) in p53-deficient models (Fig. 4b and Supplementary Fig. 2B). MLN4924 has previously been implicated in promoting JNK-mediated degradation of $\operatorname{FLIP}(\mathrm{L})$ and $\operatorname{FLIP}(\mathrm{S})^{47}$. Our results suggest that the residual FLIP(L) expression in MLN4924-treated cells is a major mechanism of resistance to this agent in p53 wildtype CRC cells.

In the metastatic disease setting ( $\mathrm{mCRC}$ ), CRC patients are currently treated with 5FU-based regimens (plus oxaliplatin, FOLFOX; or plus irinotecan, FOLFIRI); however, 5 -year overall survival rates are only $\sim 8 \%{ }^{13}$. In absence of a predictive biomarker, novel therapeutics have had modest effects as monotherapy for the treatment of mCRC. Hence, there is a critical need to develop new therapeutic approaches for mCRC. The study reported by Picco et al. ${ }^{14}$ did not find a correlation between response to MLN4924 monotherapy and clinically relevant CRC molecular drivers (KRAS mutation, $B R A F$ mutation, and microsatellite instability); however, transcriptional profiling of a cohort of mCRC tumors suggested that high-grade mucinous carcinomas could be a responsive subgroup ${ }^{14}$. Our data suggest that both p53 wild-type and particularly mutant mCRC may be responsive to MLN4924 in combination with irinotecancontaining regimens (FOLFIRI), whereas combinations with oxaliplatin (FOLFOX) would be most effective in p53 wild-type mCRC. Thus, our data support further evaluation of the second-generation proteostasis-disrupting agent MLN4924 in CRC; in particular, combinations of MLN4924 with irinotecan-containing 
chemotherapy regimens may be particularly effective in chemo-refractory p53 mutant CRC.

\section{Materials and methods Compounds}

MLN4924 [I-502] (Pevonedistat) was obtained from Boston Biochem (Cambridge, MA). SN38, oxaliplatin, and 5FU were obtained from Belfast City Hospital, Belfast Health and Social Care Trust, Belfast. Human TRAILneutralizing antibody was purchased from R\&D Systems (Minneapolis, MN).

\section{Cell lines and cell culture}

HCT116 $\mathrm{p}^{2} 3^{+/+}$and $\mathrm{p} 53^{-1-}$ cell lines were obtained from the Vogelstein Laboratory (Johns Hopkins University School of Medicine, Baltimore). LoVo shScr and shp53 were generated by transducing the parental model with retroviral pSUPER vectors expressing control or p53 short hairpin RNA under puromycin selection $(0.5 \mu \mathrm{g} / \mathrm{mL})$. HCT116 BAX/BAK DKO cells were obtained from Professor Markus Rehm (University of Stuttgart, Germany). HCT116 caspase-8 CRISPR cells were obtained from Professor Galit Lahav (Department of Systems Biology, Harvard Medical School, Boston, MA) ${ }^{48}$. All HCT116-derived cell lines were cultured in McCoy's 5A Modified Medium (ATCC, LGC Standards, Middlesex, UK) supplemented with 10\% fetal bovine serum (Invitrogen, Carlsbad, CA). LoVo cells were cultured in Dulbecco's modified Eagle's medium (ATCC, LGC Standards, Middlesex, UK) with 10\% fetal bovine serum, at $37^{\circ} \mathrm{C}$ in a humidified atmosphere of $5 \% \mathrm{CO}_{2}$. Cell lines in culture were tested at least monthly for Mycoplasma using the Lonza MycoAlert ${ }^{\mathrm{TM}}$ kit.

\section{Western blot analysis}

Whole-cell protein lysates were prepared and Western blotting was carried out as previously described ${ }^{49}$. PARP, FAS, DR5, BIM, BID, NOXA, PUMA, and procaspase-3specific antibodies were obtained from Cell Signaling Technology (Danvers, MA). Cullin-3-specific antibody was obtained from BD Biosciences (Santa Jose, CA); p53- and p21-specific antibodies were obtained from Santa Cruz Technologies (Dallas, TX); FLIP-specific antibody (NF6) was obtained from Adipogen (San Diego, CA). Caspase-8 antibody was from Enzo Life Sciences (Farmingdale, NY). Secondary horseradish peroxidase-conjugated antibodies from Cell Signaling Technology (Danvers, MA) were used for detection on a G-Box digital developer (Syngene Cambridge, UK). Antibody catalog numbers are listed in Supplementary Table S4.

\section{Flow cytometry}

Detection of cell-surface DR5 and Fas expression was conducted using the BD Accuri C6 flow cytometer, with analyses completed on the Accuri C6 PLUS software (BD
Biosciences, San Diego, CA), and cells stained using Phycoerythrin-conjugated anti-DR5 or anti-FAS antibody compared with an isotype control antibody (IgG) (Biolegend, San Diego, CA). Annexin-V/Propidium Iodide flow cytometry was carried out on a BD LSRII flow cytometer (BD Biosciences, San Diego, CA) using fluorescein isothiocyanate (FITC)-tagged Annexin-V (BD Biosciences) and Propidium Iodide (Sigma-Aldrich, MO). Loss of mitochondrial outer membrane potential was quantified following staining with $25 \mathrm{nM}$ Tetramethylrhodamine ethyl ester (Sigma-Aldrich, MO) for $15 \mathrm{~min}$ prior to flow cytometric analyses on the BD Accuri C6 flow cytometer.

\section{High-content microscopy}

Cells were seeded into a 96-well glass-bottomed plate (Cellvis) and left to adhere overnight. After treatments, cells were incubated with $10 \times$ Annexin-V Binding Buffer, 1:1000 FITC Annexin-V (BD Pharmingen, San Diego, $\mathrm{CA}$ ), $0.33 \mu \mathrm{g} / \mathrm{mL}$ Propidium Iodide (Sigma-Aldrich, MO), and $1.33 \mu \mathrm{g} / \mathrm{mL}$ Hoechst 33342 (Thermo Fisher Scientific, Waltham, MA) for $20 \mathrm{~min}$ at room temperature. The plate was analyzed on the ArrayScanTM XTI HCA Reader, integrated with the CrESTTM X-LightTM Confocal Scan Head (Thermo Fisher Scientific, Waltham, MA).

\section{siRNA transfections}

All siRNAs, apart from those targeting FLIP (Eurofins Scientific, Luxembourg), were obtained from Dharmacon (Chicago, IL) and transfections carried out using Lipofectamine RNAiMAX (Life Technologies, Carlsbad, CA), as previously described ${ }^{50}$.

\section{Cell death siRNA library screen}

A Dharmacon siGENOME SMARTpool siRNA library was generated to target 41 cell death regulators. siRNA duplexes were seeded onto 384-well source plates, which were stored at $-20^{\circ} \mathrm{C}$ until required. Using an automatic acoustic liquid handler, the Labcyte ECHO (Labcyte, San Jose, CA), siRNAs were transferred from the source plate to a 384-well destination plate. Lipofectamine RNAiMAX Transfection reagent was mixed in a 1:167 ratio with OptiMEM reduced-serum media (Thermo Fisher Scientific, Waltham, MA) and $10 \mu \mathrm{L}$ was added to each well and incubated for $20 \mathrm{~min}$ to allow for liposome formation. Nine hundred cells were seeded into each well, each containing a final concentration of $10 \mathrm{nM}$ siRNA, and plates were incubated overnight to allow cells to adhere and transfection to occur. The following day, the transfection mix was removed and $50 \mu \mathrm{L}$ relevant antibioticfree media was added containing appropriate MLN4924 concentrations and incubated for the required time. Transfection conditions, cell seeding densities, drug concentrations, and time points were optimized in pilot experiments with specific transfection controls 
(untransfected, non-targeting siRNA, and PLK1 siRNA) used to confirm adequate transfection efficiency.

\section{Caspase and cell-viability assays}

Caspase activity was measured using Caspase-Glo® assay reagents and cell viability assessed by Cell Titer-Glo® (Promega, Madison, WI).

\section{Clonogenic survival assay}

Cells were seeded at a density of 500 cells per well and treated for $24 \mathrm{~h}$ before media replacement. Following 7-10 days, colony formation was assessed via Crystal Violet staining and quantified by resuspension of the dye absorbed by the colonies using $1 \mathrm{M}$ sodium citrate:50\% ethanol solution, with absorbance measured at $570 \mathrm{~nm}$ on the Biotrak II microplate reader.

\section{Statistical analysis}

Statistical significance was calculated from distinct technical replicates $(n \geq 3)$, either by Student's $T$-test (twotailed, two-sample equal variance on unpaired data) or two-way analysis of variance in GraphPad Prism 8. Graphs were plotted as means with error bars represented as SEM; statistical significance was denoted as follows: ${ }^{* * * * *} p<$ $0.0001, * * * 0<0.001, * * p<0.01, \quad * p<0.05$, ns $=p>0.05$. Experimental phenotypes were confirmed in at least three independent experiments.

SI was a statistical method utilized to interpret raw data obtained from the cell death siRNA screen. SI estimates the combined influence of siRNA knockdown on drug sensitivity and the individual drug and siRNA effects.

$\mathrm{SI}=\left(\mathrm{Rc} / \mathrm{Cc}^{*} \mathrm{Cd} / \mathrm{Cc}\right)-(\mathrm{Rd} / \mathrm{Cc})$, where

Rc: average viability in untreated wells transfected with siRNA

Rd: average viability in drug-treated wells with siRNA

Cc: average viability in untreated wells with control siRNA

$\mathrm{Cd}$ : average viability in drug-treated wells with control siRNA

The SI value ranges from -1 to 1 , with positive values indicating a sensitizing effect and negative values indicating an antagonizing effect.

\footnotetext{
Acknowledgements

This work was funded by grants from the Northern Ireland Department for the Economy (NI DfE) studentship (J.F.) plus funding from CRUK Program grant C11884/A24367 (PI: D.B.L.; Cl: S.S.M.) and Experimental Cancer Medicine Centre (ECMC) grant C36697/A25176. We thank Professor Bert Vogelstein (Johns Hopkins University School of Medicine, Baltimore) for kindly gifting the HCT116 $\mathrm{p} 53^{+/+}$and $\mathrm{p}^{2} 3^{-/-}$cell lines, Professor Markus Rehm (University of Stuttgart, Germany) for supplying the HCT116 BAX/BAK DKO cells, and Professor Galit Lahav (Department of Systems Biology, Harvard Medical School, Boston, MA) for providing the HCT116 caspase-8 CRISPR cells.
}

\section{Author contributions}

J.F.: conceptualization, methodology, validation, formal analysis, investigation, writing (original draft), and visualization. M.E.-F.: investigation. C. Hamilton: investigation. C. Holohan: conceptualization, resources, and supervision. N.C.: methodology and investigation. A.M.: resources and methodology. J.Z.R.: resources and methodology. M.W.: investigation and methodology. S.M.D.: resources and methodology. D.B.L.: conceptualization, methodology, validation, formal analysis, writing (original draft), visualization, supervision, project administration, and funding acquisition. V.C.: conceptualization, methodology, validation, formal analysis, writing (original draft), visualization, supervision, project administration, and funding acquisition.

\section{Data availability}

The data supporting the findings of the study are available from the corresponding author on reasonable request.

\section{Conflict of interest}

The authors declare that they have no conflict of interest.

\section{Publisher's note}

Springer Nature remains neutral with regard to jurisdictional claims in published maps and institutional affiliations.

The online version of this article (https://doi.org/10.1038/s41420-020-00296-w) contains supplementary material, which is available to authorized users.

Received: 1 May 2020 Revised: 15 June 2020 Accepted: 30 June 2020 Published online: 21 July 2020

\section{References}

1. Nalepa, G., Rolfe, M. \& Harper, J. W. Drug discovery in the ubiquitinproteasome system. Nat. Rev. Drug Discov. 5, 596-613 (2006).

2. Huang, Z. et al. Efficacy of therapy with bortezomib in solid tumors: A review based on 32 clinical trials. Future Oncol. 10, 1795-1807 (2014).

3. Hori, T. et al. Covalent modification of all members of human cullin family proteins by NEDD8. Oncogene 18, 6829-6834 (1999).

4. Jones, J. et al. A targeted proteomic analysis of the ubiquitin-like modifier nedd8 and associated proteins. J. Proteome Res. 7, 1274-1287 (2008).

5. Brownell, J. E. et al. Substrate-assisted inhibition of ubiquitin-like protein-activating enzymes: the NEDD8 E1 inhibitor MLN4924 forms a NEDD8-AMP mimetic in situ. Mol. Cell 37, 102-111 (2010).

6. Bennett, E. J., Rush, J., Gygi, S. P. \& Harper, J. W. Dynamics of cullin-RING ubiquitin ligase network revealed by systematic quantitative proteomics. Cell 143, 951-965 (2010).

7. Watson, I. R., Irwin, M. S. \& Ohh, M. NEDD8 pathways in cancer, sine quibus non. Cancer Cell 19, 168-176 (2011).

8. Zhou, L., Jiang, Y., Luo, Q., Li, L. \& Jia, L. Neddylation: a novel modulator of the tumor microenvironment. Mol. Cancer 18, 77 (2019).

9. Soucy, T. A. et al. An inhibitor of NEDD8-activating enzyme as a new approach to treat cancer. Nature 458, 732-736 (2009).

10. ClinicalTrials.gov. Available at: https://clinicaltrials.gov/ct2/results? cond $=\&$ term $=M L N 4924 \&$ cntry $=\&$ state $=\&$ city $=\& d i s t=($ accessed 1 April 2020).

11. Bray, F. et al. Global cancer statistics 2018: GLOBOCAN estimates of incidence and mortality worldwide for 36 cancers in 185 countries. CA Cancer J. Clin. $\mathbf{6 8}$, 394-424 (2018)

12. Siegel, R. L. et al. Colorectal cancer statistics, 2017. CA Cancer J. Clin. 67 , 177-193 (2017)

13. Insua, Y. V. et al. Predicting outcome and therapy response in $m C R C$ patients using an indirect method for CTCs detection by a multigene expression panel: a multicentric prospective validation study. Int. J. Mol. Sci. 18, 1265 (2017).

14. Picco, G.et al. Efficacy of NEDD8 pathway inhibition in preclinical models of poorly differentiated, clinically aggressive colorectal cancer. J. Natl. Cancer Inst. 109, djw209 (2017).

15. Tong, S. et al. MLN4924 (Pevonedistat), a protein neddylation inhibitor, suppresses proliferation and migration of human clear cell renal cell carcinoma. Sci. Rep. 7, 5599 (2017).

16. Wei, D. et al. Radiosensitization of human pancreatic cancer cells by MLN4924 an investigational NEDD8-activating enzyme inhibitor. Cancer Res. 72, 282-293 (2012). 
17. Li, H. et al. Inhibition of Neddylation modification sensitizes pancreatic cancer cells to Gemcitabine. Neoplasia (U. S.) 19, 509-518 (2017).

18. Wang, J. et al. Targeting neddylation pathway with MLN4924 (Pevonedistat) induces NOXA-dependent apoptosis in renal cell carcinoma. Biochem. Biophys. Res. Commun. 490, 1183-1188 (2017).

19. Chen, P. et al. Neddylation inhibition activates the extrinsic apoptosis pathway through ATF4-CHOP-DR5 axis in human esophageal cancer cells. Clin. Cancer Res. 22, 4145-4157 (2016).

20. Barretina, J. et al. Addendum: The Cancer Cell Line Encyclopedia enables predictive modelling of anticancer drug sensitivity. Nature 565, E5-E6 (2019).

21. lorio, F. et al. A landscape of pharmacogenomic interactions in cancer. Cell 166, 740-754 (2016)

22. Yang, W. et al. Genomics of Drug Sensitivity in Cancer (GDSC): a resource for therapeutic biomarker discovery in cancer cells. Nucleic Acids Res. 41, 955-961 (2013).

23. Ritchie, M. E. et al. Limma powers differential expression analyses for RNAsequencing and microarray studies. Nucleic Acids Res. 43, e47 (2015).

24. Kuleshov, M. V. et al. Enrichr: a comprehensive gene set enrichment analysis web server 2016 update. Nucleic Acids Res. 44, W90-W97 (2016).

25. Mikhailov, V. et al. Association of Bax and Bak homo-oligomers in mitochondria: Bax requirement for Bak reorganization and cytochrome c release. J. Biol. Chem. 278, 5367-5376 (2003).

26. Li, H., Zhu, H., Xu, C. \& Yuan, J. Cleavage of BID by caspase 8 mediates the mitochondrial damage in the Fas pathway of apoptosis. Cell 94, 491-501 (1998).

27. Takahashi, N. et al. RIPK1 ensures intestinal homeostasis by protecting the epithelium against apoptosis. Nature 513, 95-99 (2014).

28. Ka-Ming, F., Luz, N. F. \& Moriwaki, K. Programmed necrosis in the cross talk of cell death and inflammation. Annu. Rev. Immunol. 79, 106 (2015).

29. Carson, R. et al. HDAC inhibition overcomes acute resistance to MEK inhibition in BRAF-mutant colorectal cancer by downregulation of c-FLIPL. Clin. Cancer Res. 21, 3230-3240 (2015).

30. Takimoto, R. \& El-Deiry, W. S. Wild-type p53 transactivates the KILLER/DR5 gene through an intronic sequence-specific DNA-binding site. Oncogene 19 1735-1743 (2000).

31. Toshiyuki, M. \& Reed, J. C. Tumor suppressor p53 is a direct transcriptional activator of the human bax gene. Cell 80, 293-299 (1995).

32. Hudson, C. D., Morris, P. J., Latchman, D. S. \& Budhram-Mahadeo, V. S. Brn-3a transcription factor blocks p53-mediated activation of proapoptotic target genes Noxa and Bax in vitro and in vivo to determine cell fate. J. Biol. Chem. 290, 887 (2005)

33. Hanahan, D. \& Weinberg, R. A. The hallmarks of cancer. Cell 100, 57-70 (2000).

34. Wan, J., Zhu, J., Li, G. \& Zhang, Z. Radiosensitization of human colorectal cancer cells by MLN4924: an inhibitor of NEDD8-activating enzyme. Technol. Cancer Res. Treat. 15, 1-8 (2015).
35. Lin, J. J., Milhollen, M. A., Smith, P. G., Narayanan, U. \& Dutta, A. NEDD8 targeting drug MLN4924 elicits DNA rereplication by stabilizing Cdt1 in S phase, triggering checkpoint activation, apoptosis, and senescence in cancer cells. Cancer Res. 70, 10310-10320 (2010).

36. Bailly, A. et al. The NEDD8 inhibitor MLN4924 increases the size of the nucleolus and activates p53 through the ribosomal-Mdm2 pathway. Oncogene 35, 415-426 (2016).

37. Liu, X. et al. NEDD8-activating enzyme inhibitor, MLN4924 (Pevonedistat) induces NOXA-dependent apoptosis through up-regulation of ATF-4. Biochem. Biophys. Res. Commun. 488, 1-5 (2017).

38. Chipuk, J. E. et al. Direct activation of Bax by p53 mediates mitochondrial membrane permeabilization and apoptosis. Science 303, 1010-1014, https:// doi.org/10.1126/science.1092734 (2004).

39. Leu, J. I. J., Dumont, P., Hafey, M., Murphy, M. E. \& George, D. L. Mitochondrial p53 activates Bak and causes disruption of a Bak-Mcl1 complex. Nat. Cell Biol. 6, 443-450 (2004)

40. McLaughlin, K. A. et al. FLIP: a targetable mediator of resistance to radiation in non-small cell lung cancer. Mol. Cancer Ther. 15, 2432-2441 (2016).

41. Lee, S., Yoon, C. Y., Byun, S.-S., Lee, E. \& Lee, S. E. The role of c-FLIP in cisplatin resistance of human bladder cancer cells. J. Urol. 189, 2327-2334 (2013).

42. Kamarajan, P., Sun, N.-K. \& Chao, C. C.-K. Up-regulation of FLIP in cisplatinselected HeLa cells causes cross-resistance to CD95/Fas death signalling. Biochem. J. 376, 253-260 (2003).

43. Krueger, A., Schmitz, I., Baumann, S., Krammer, P. H. \& Kirchhoff, S. Cellular FLICE-inhibitory protein splice variants inhibit different steps of caspase-8 activation at the CD95 death-inducing signaling complex. J. Biol. Chem. $\mathbf{2 7 6}$ 20633-20640 (2001)

44. Wilson, T. R. et al. Procaspase 8 overexpression in non-small-cell lung cancer promotes apoptosis induced by FLIP silencing. Cell Death Differ. 16, 1352-1361 (2009).

45. Lu, M. et al. Opposing unfolded-protein-response signals converge on death receptor 5 to control apoptosis. Science 345, 98-101 (2014).

46. Lam, M., Marsters, S., Ashkenazi, A. \& Walter, P. Misfolded proteins bind and activate death receptor 5 to trigger apoptosis during unresolved endoplasmic reticulum stress. Elife 9, e52291 (2020).

47. Zhao, L., Yue, P., Lonial, S., Khuri, F. R. \& Sun, S.-Y. The NEDD8-activating enzyme inhibitor, MLN4924, cooperates with TRAIL to augment apoptosis through facilitating c-FLIP degradation in head and neck cancer cells. Mol. Cancer Ther 10, 2415-2425 (2011)

48. Paek, A. L., Liu, J. C., Loewer, A., Forrester, W. C. \& Lahav, G. Cell-to-cell variation in p53 dynamics leads to fractional killing. Cell 65, 631-642 (2016).

49. Longley, D. B. D. et al. c-FLIP inhibits chemotherapy-induced colorectal cancer cell death. Oncogene 25, 838-848 (2006).

50. Crawford, N. et al. SAHA overcomes FLIP-mediated inhibition of SMAC mimetic-induced apoptosis in mesothelioma. Cell Death Dis. 4, e733-11 (2013). 